\title{
ROTATION, ACTIVITY AND LITHIUM DEPLETION IN THE HYADES LATE MAIN SEQUENCE
}

\author{
R. Rebolo and J.E. Beckman, \\ Instituto de Astrofisica de Canarias, \\ 38200 - La Laguna, \\ Tenerife, \\ Spain.
}

\begin{abstract}
Selecting stars with previously measured or estimated rotational periods we measured $\mathrm{Li}$ equivalent widths in 8 stars of the Hyades late main sequence. Combining our data with literature values, we derive a homogeneous set of $L i$ abundances. Plotting $\log N(L i)$ against Rossby number we can separate the effects of temperature and rotation. We find for stars later than F8 increased depletion with longer period, at constant surface temperature. Discounting, as improbable, spurious abundances due to surface activity effects, we give a tentative view of the implications of these results for stellar modelling.
\end{abstract}

\section{INTRODUCTION}

A star's present $L i$ abundance depends on its initial value, $L i$ or and on the mechanisms which transport $\mathrm{Li}$ down to its nuclear burning depth. The wellknown monotonic relation $\log N(L i) v$. Teff between $F 8$ and $K 0$ in clusters is due to the increase in the convection zone depth with decreasing stellar mass. Use of $\mathrm{Li}$ to determine age or to probe interiors is inhibited by our limited knowledge of the processes transporting $\mathrm{Li}$ across the "gap" between the base of the convection zone and the nuclear burning zone. Among recent models which agree quite well with the log $N(L i) v$. Teff observations in the Hyades is that of Baglin et al. (1985) using a turbulent diffusion mechanism proposed by Zahn (1984); (see also Baglin et al. (1987), this volume). Here we use the Hyades, where many rotation periods are measured to probe rotation effects on Li depletion.

\section{LITHIUM DATA}

We obtained spectra of 8 Hyades MS stars, at the Cassegrain ID spectrograph of the $2.5 \mathrm{~m}$ Isaac Newton Telescope, La Palma, with spectral resolution $\lambda / \Delta \lambda$ $\sim 2 \times 10^{4}$, and $S: N$ ratios between 150 and 200 at $\lambda 6708 \mathrm{~A}$, using a CCD detector. To these we added literature $\mathrm{Li}$ abundances (Cayrel et al., 1984) and equivalent widths, (Duncan and Jones 1983; Zappala, 1972). We analyzed data using the same Teff and log $\mathrm{g}$ calibrations, and the same model atmospheres 
as Cayrel et al. (1984). Relative Li abundance errors are less than 0.10 dex.

\section{LITHIUM v. PERIOD AND ROSSBY NUMBER}

Periods were either from rotational modulation of the Strömgren $b, y$ colours (Lockwood et al., 1984) with errors $\pm 3 \%$, or from $H$ and $K$ fluxes by Duncan et al. (1984) using the relation of Noyes et al. (1984) between these and the period, which has errors of $\pm 20 \%$. In Fig. 1a we plot $\log N(L i) v$. period $P$, and in Fig. 1b we show log $N(\mathrm{Li})$ v. Teff. The two curves are strikingly similar, with a little more scatter in the former. This result is not sucprising, given the role of deeper convection zones in destroying $\mathrm{Li}$, and also in facilitating magnetic braking.

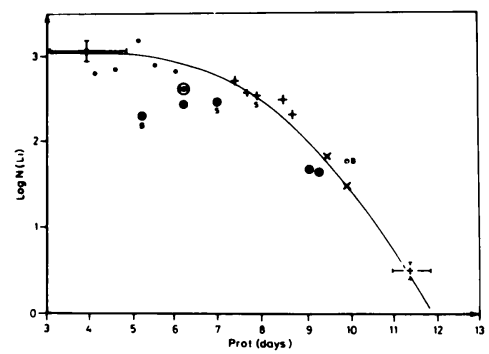

Fig 1a: Rlot of Li abundance v. period for Hyades main sequence stars. Key:

- Li data from Duncan and Jones (1983) or Zappala (1972). Periods predicted from $\mathrm{Ca}$ fluxes.

+ Li data from Cayrel (1984). Period measurements from Lockwood et al. (1984).

$\times$ Li from Cayrel. Periods estimated from Ca Fluxes.

- Li data from this work. Periods

from Lockwood et al. (1984). L Li from this work. Periods from Ca fluxes.

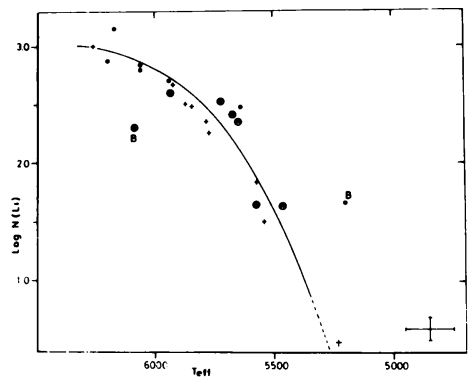

Fig $1 \mathrm{~b}: \mathrm{Li}$ abundance v. temperature for the same stars as in Fig 1a.

In Fig. 2 we plot log $N(L i)$ v. Rossby number $N_{R} P P / \tau_{c}$ where $\tau_{c}$ is the convective zone turnover time (see Noyes et al., 1984). Note that this plot is a dispersed family of curves, in contrast to the monotonic dependence of $\mathrm{Ca} H$ and $\mathrm{K}$ emission on $\mathrm{N}_{\mathrm{R}}$ in Noyes et al. (1984). Note also that along each line of constant $B-V, L i$ is more depleted at longer period. This feature implies a rotation effect independent of Teff or mass.

A note of caution: activity effects accompanying rotation could just possibly produce the curves in Fig. 2. The presence of sunspots could raise the apparent $\mathrm{Li}$ abundance (Giampapa, 1984); plage cover, on the other hand, would tend to reduce it. We believe such effects to be insignificant, because no modulation of $\mathrm{Li}$ eq. width has been detected even in stars rotating more rapidly that this sample (see comments after Soderbtom in this volume), and 
because the colour "anomaly" in $B-V$ against $V-K$ (see Campbell, 1984) is very small for our stars.

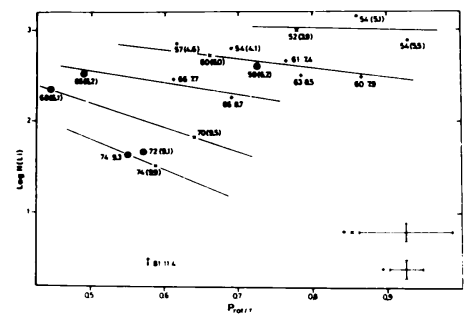

Fig. 2: Li abundance versus Rossby number for the Hyades stars. Trend lines indicate objects of similar $(B-V)$ values. Integer numbers are $(B-V) \times 100$. Decimal numbers are periods (brackets estimated from $\mathrm{Ca}$ fluxes; without brackets measured directly). Symbols are as in Fig 1 .

\section{ROTATION AND LI DEPLETION}

There are three possible causes for the different periods $P$ exhibited by the Hyades main sequence stars. These are: (a) They started with different initial periods $P_{0}$, or (b) they started with identical values of $P_{0}$ but have different ages, or (c) they have been differentially braked by different "frozen in" initial magentic fields. We can discount (c) because the timescale for poloidal field regeneration is very short, on the order of the stellar magnetic cycle time, i.e. a few years (Durney et al., 1981) which implies that any frozen-in fields would not last longer than a few cycles at most. Whilst we cannot rule out (b) it is difficult to sustain numerically. For stars with $(B-V)=0.66$, there is an apparent dispersion in period of $40 \%$ which would correspond to an age spread of $80 \%$ if the braking were according to the empirical law of Skumanich (1972). This large age range is not easily compatible with the Hyades H-R diagram. However, given the uncertainty in the estimates of $P$, especially the indirect estimates, we would not be justified in ruling out differential age effects in the cluster.

Option (a) is a very probable cause of the present dispersion in P. It is not easy, however, to reconcile this as the principal cause with the dependence of $\mathrm{Li}$ abundance on Rossby number shown. in Fig. 2. If a slower rotation now means that the star has always rotated more slowly, then slower rotators deplete Li more rapidly (for equal mass) than more rapid rotators. This can be consistent with models where the cause of $\mathrm{Li}$ depletion is rotation-driven turbulent diffusion (e.g. Scbatzman, 1977; Baglin et al., 1985) only by assuming that the rotation also tends to inhibit the depth of the subphotospheric convective zone (or of the overshooting) especially for small values of $P$. We must therefore suspend final physical judgement until better data, especially on the periads, but also more $\mathrm{Li}$ abundances, are available for the Hyades.

References

Baglin, A., Morel, P.J., Schatzman, E.: 1985, Astron. Astrophys 149, 309. Campbell, B.: 1984, Astrophys. J. 283, 209.

Cayrel, R., Cayrel de Strobel, G., Campbell, B.: 1984, Astrophys. J. 283, 205.

Duncan, D.K., Baliunas, S.L., Noyes, R.W., Vaughan, A.H., Frazer, M.J., Canning, H.: 1984, PASP, 96, 707.

Duncan, D.K., Jones, B.: 1983, Astrophys. J. $271,663$.

Durney, B.R., Mihalas, D., Robinson, R.: 1981, PASP 93, 537.

Giampapa, M.S.: 1984, Astrophys. J. 277, 235. 
Lockwood, G.W., Thompson, D., Radick, R.R., Osborn, W.H., Baggett, W.E., Duncan, D.K., Hartmann, L.W.: 1984, PASP 96, 714.

Noyes, R.W., Hartmann, L.W., Baliunas, S.L., Duncan, D.K., Vaughan, A.H.: 1984, Astrophys. J. 279, 763.

Schatzman, E.: 1977, Astron. Astrophys. 56, 211.

Skumanich, A.: 1972, Astrophys. J. 171, 565.

Vaughan, A.H., Baliunas, S.L., Middelkoop, R., Hartmann, L.W., Mihalas, D., Noyes, R.W., Preston, G.W.: 1981, Astrophys. J. 250, 276.

Zahn, J.P.: 1984, In Physics of Early-Type Stars, Eds. B. Hauck, A. Maeder, Geneva Obs. Press.

Zappala, R.R.: 1972, Astrophys. J. 172, 57.

\section{DIscussion}

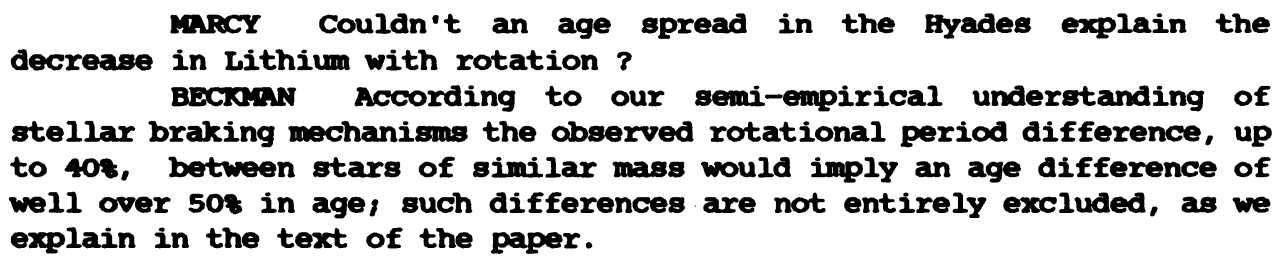

